Tropical Journal of Pharmaceutical Research October 2020; 19 (10): 2079-2084

ISSN: $1596-5996$ (print); 1596-9827 (electronic) (C) Pharmacotherapy Group, Faculty of Pharmacy, University of Benin, Benin City, 300001 Nigeria.

\title{
Downregulation of miR-451a in plasma may promote prothrombin expression and contribute to high blood coagulation state in gestational diabetes mellitus
}

\author{
Min Qiu1, Zhimin Shangguan², Hao Zhang ${ }^{3 *}$ \\ ${ }^{1}$ Department of Gynaecology and Obstetrics, ${ }^{2}$ Department of Blood Transfusion, The Third Affiliated Hospital of Soochow \\ University, Changzhou City, Jiangsu Province 213003, ${ }^{3}$ Department of Gynaecology, Obstetrics and Gynecology Hospital \\ Affiliated of Fudan University, Shanghai 200021, China
}

*For correspondence: Email: zhanghao_hww@163.com; Tel: +86-21-33189900

Sent for review: 8 July 2020

Revised accepted: 27 September 2020

\begin{abstract}
Purpose: To investigate $F 2$ and miR-451a levels in gestational diabetes mellitus (GDM) patients, evaluate the potential role of miR-451a in GDM, and to explore its possible downstream targets. Methods: Thromboelastography (TEG) analysis was conducted to analyze coagulation differences between the GDM and control groups. Quantitative polymerase chain reaction (qPCR) and immunoblot were performed to analyze miR-451a and F2 levels after exposure to high glucose and miR-451a mimics or an miR-451a inhibitor. Luciferase assay was used to explore the potential regulatory mechanism.

Results: Enhancement of the patients' blood coagulation ability and down-regulated miR-451a expression levels occurred in the plasma of GDM patients. The data further show that hepatocytes exposed to high glucose reduced miR-451a levels and enhanced F2 expression. The overexpression of miR-451a inhibited the expression of F2.

Conclusion: MiR-451a may serve as a promising marker for hypercoagulable state GDM patients

Keywords: Gestational diabetes mellitus (GDM), MiR-451a, F2, Hypercoagulable state, Prothrombin, Thromboelastography (TEG)

This is an Open Access article that uses a funding model which does not charge readers or their institutions for access and distributed under the terms of the Creative Commons Attribution License (http://creativecommons.org/licenses/by/4.0) and the Budapest Open Access Initiative (http://www.budapestopenaccessinitiative.org/read), which permit unrestricted use, distribution, and reproduction in any medium, provided the original work is properly credited.

Tropical Journal of Pharmaceutical Research is indexed by Science Citation Index (SciSearch), Scopus, International Pharmaceutical Abstract, Chemical Abstracts, Embase, Index Copernicus, EBSCO, African Index Medicus, JournalSeek, Journal Citation Reports/Science Edition, Directory of Open Access Journals (DOAJ), African Journal Online, Bioline International, Open-J-Gate and Pharmacy Abstracts
\end{abstract}

\section{INTRODUCTION}

Gestational diabetes mellitus (GDM) is a common complication in pregnancy [1] that may lead to embryo defects, abortion, abnormal fetal development, and premature delivery [2]. Blood coagulation enhancement is common in women in the third trimester of pregnancy [3]. Thromboelastography (TEG) could reveal the specific characteristics of hypercoagulability of blood during pregnancy. Hypercoagulability of blood is harmful for human bodies which can induce venous thrombosis in pregnant and parturient women [1]. In severe cases, repeated abortion and premature delivery may occur, which may result in maternal deaths [4]. Pregnant women may experience blood coagulation enhancement. Similarly, some diabetics also have blood coagulation enhancement. 
Prothrombin is a serum clotting factor that is synthesized in the liver and dependent on vitamin $\mathrm{K}$ [5]. Factor $\mathrm{Xa}$ complex acts on prothrombin, breaking the two bonds on prothrombin, forming thrombin $\mathrm{F} 1$ and $\mathrm{F} 2(\mathrm{~F} 1+2)$ [5]. Thrombin $\mathrm{F} 1+2$ reflects the activity of factor Xa complex and is a specific molecular marker for the activation of prothrombin [6]. Recent studies have found that elevated plasma $\mathrm{F} 1+2$ levels are associated with the presence of atherosclerosis and the risk of clinically symptomatic cardiovascular disease [6]. The expression of prothrombin is closely related to hyperglycemia [7]. MicroRNA, with lengths of 1822 bp [8] interacts with the 3'-non-transcriptional region of the genes [8]. Several studies have found that microRNA levels were changed in patients with GDM [9]. For example, upregulation of miR-503 in GDM led to impairment of endothelial cell function [10]. MiR-222 regulates the expression of ER, thereby regulating insulin resistance in GDM [11]. In addition, studies have also shown miRNAs have important regulatory effects on blood coagulation [9]. MiR-451a expression is down-regulated in the placental exosomes of patients with GDM [12]. Herein, the data showed that the expression level of miR-451a in the plasma of GDM patients was down-regulated while blood coagulation was enhanced. Therefore, circulation of miR-451a was speculated to be closely related to the blood coagulation ability of GDM patients.

\section{EXPERIMENTAL}

\section{Thromboelastography}

Ethical approval was obtained from the Ethics Committee of the Third Affiliated Hospital of Soochow University. A total of 44 Gestational diabetes mellitus (GDM) patients and 32 normal pregnant women underwent thromboelastography treated at The Third Affiliated Hospital of Soochow University using TEG @ 5000 Thromboelastograph Hemostasis Analyzer System (Haemonetics, Braintree, MA, USA). Blood was retrieved with the two-syringe method. Subsequently blood was citrated with $0.2 \mathrm{M}$ solution of calcium chloride and added to a warmed cuvette. Thromboelastography parameters, including time $r$ (minutes), time $\mathrm{k}$ (minutes), a angle (degrees), and maximum amplitude (millimeters) were recorded.

\section{Cell culture and transfection}

The HepG2 cell line was purchased from the American Type Culture Collection and incubated in Dulbecco's modified essential medium supplemented with $10 \%$ fetal bovine serum (Gibco, CA, USA). For glucose stimulation, HepG2 cells were cultured in complete medium supplemented with $50 \mathrm{mM}$ D-glucose for $72 \mathrm{~h}$. Synthetic miR-451a mimics, miRNA mimic negative control, miR-451a inhibitor, and negative control RNA inhibitor were purchased from GenePharma (Shanghai, China). Transfection was conducted using Lipofectamine 3000 Reagent (Thermo Fisher Scientific, Waltham, MA, USA) according to the manufacturer's protocols.

\section{Enzyme-linked immunosorbent assay (ELISA)}

According to the manufacturer's instructions, blood was analyzed to determine the levels of Thrombin Activatable Fibrinolysis Inhibitor (TAFI), a marker of impaired endogenous fibrinolysis, with an Imuclone Total TAFI ELISA kit (BioMedica Diagnostics, Windsor, NS, Canada), and prothrombin fragment $1+2(\mathrm{~F} 1+2)$ with a human Prothrombin fragment 1+2 ELISA kit (Wuhan Fine Biotech Co., Wuhan City, Hubei, China).

\section{Quantitative polymerase chain reaction (qPCR)}

Total RNA was extracted using TRIzol (Invitrogen, Waltham, MA, USA) according to the manufacturer's protocol. For miR-451a detection, total RNA was transcribed to cDNA using the miRNA $1^{\text {st }}$-strand cDNA synthesis kit (Vazyme, Nanjing, China) and further subjected to qPCR using a miRNA SYBR qPCR Master Mix kit (Vazyme). U6 snRNA was used as an internal control for miR-451a. The sequences of primers used in this study were Has-miR-451a forward: 5'-

ACACTCCAGCTGGGAAACCGTTACCATTAC3' Has-miR-451a reverse: 5'-CTCAACTGGTGT CGTGGAGTCGGCAATTCAGTTGAGCTTACAG -3', U6 forward: 5'-CGCTTCGGCAGCACATAT AC-3', U6 reverse: 5'-TTCACGAATTTGCGTGT CATC-3', prothrombin forward: 5'-CTAGCACC TAGGACAAGCCTGATGAAGGGAAAC-3', prothrombin reverse: 5'-CACTCGGATCCCCTCC CACGTAGCTGGGACTA-3', GAPDH forward: 5'CGACCACTTTGTCAAGCTCA-3', GAPDH reverse 5'-GGTTGAGCACAGGGTACTTTATT3'.

\section{Western blotting assay}

Total proteins were lysed with RIPA (Cell Signaling, Danvers, MA, USA) and subjected to SDS-PAGE. The proteins were transferred onto PVDF membranes, which were immersed in $5 \%$ BSA in TBST. The membranes were 
subsequently incubated with target-specific antibodies. Human prothrombin polyclonal antibody, which recognizes the $F 1$ portion of prothrombin (1:1000, aa 130-179, and $\beta$-actin (1:10000, ab8226), Nrf2 (1:1000, ab62352), NQO1 (1:1000, ab80588) and NLRP3 (1:1000, ab263899) were purchased from Abcam (Cambridge, UK).The membranes were then incubated with HRP-conjugated goat anti-mouse and anti-rabbit secondary antibodies (1:5000; ab6789 and ab6721, respectively; Abcam) for 45 min at room temperature. Signals were detected using an ECL kit (Novex ${ }^{\mathrm{TM}} \mathrm{ECL}$ Chemiluminescent Substrate Reagent kit; Thermo Fisher Scientific) following the manufacturer's instructions.

\section{Cloning and reporter assays}

The 3' UTR of prothrombin mRNA was amplified with specific primers and inserted into Xhol and Notl sites of the pGL3 plasmid downstream of the Renilla open reading frame. The sequence of ACGGUU in the 3' UTR of F2 was mutated into GCCAAA. Mutagenesis in the 3' UTR of prothrombin was induced using the Site-Directed Mutagenesis Kit (Agilent Technologies, Santa Clara, CA, USA) and was verified by sequencing. After co-transfection of NC mimic+pGL3-F2-WT, miR-451a mimic+pGL3-F2-WT, NC mimic+pGL3-F2-MUT, and miR-451a mimic+pGL3-F2-MUT using Lipofectamine 2000 in complete medium, the cells were harvested and a dual-luciferase assay kit (Promega, Madison, WI, USA) was used to measure reporter Renilla and firefly luciferase activities in accordance with the manufacturer's protocols.

\section{Statistical analysis}

The data were expressed as mean $\pm S D$ and analyzed using GraphPad Prism (San Diego, CA, USA) (version 5.04). $P<0.05$ was considered as statistically significant.

\section{RESULTS}

\section{GDM patients exhibited enhanced blood coagulation and reduced miR-451a levels}

To reveal the differences in coagulation function between GDM patients and control subjects, we performed thromboelastography (TEG) measurements. As shown in Figure 1A, the mean values of time $R$ and time $K$ were reduced in the GDM group, while the $\alpha$ angle and maximum amplitude were low in healthy, pregnant women (Figure $1 \mathrm{~A}$ ). These results suggest that, as compared with the control group, GDM patients differed in the degree of fibrin formation, the state of dissolution, the firmness of coagulation, and the strength of elasticity. TAFI was reduced in GDM patients, and prothrombin fragment $1+2(F 1+2)$ levels were elevated in the GDM group (Figure $1 \mathrm{~B}$ ). qPCR analysis showed a relatively lower level of miR451a in the GDM group (Figure 1C). Thus, the enhanced coagulation function and reduced miR451 a levels were revealed in GDM patients.

A
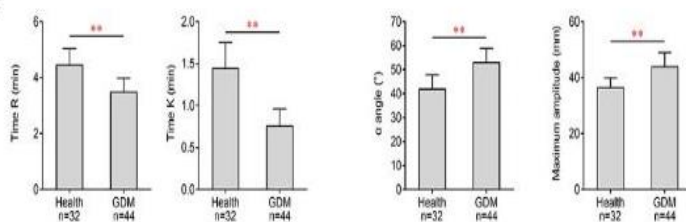

B
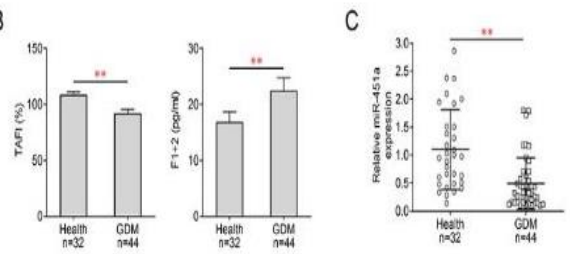

Figure 1: GDM patients showed enhanced coagulation degree and reduced miR-451a levels. (A) Evaluated thromboelastography parameters assessed were time $\mathrm{R}$, time $\mathrm{K}, \alpha$ angle, and maximum amplitude in the healthy and GDM groups. (B) TAFI and F1+2 levels were assessed in the GDM and control groups using ELISA assays. (C) Relative miR-451a levels in healthy and GDM groups. ${ }^{* *} P<0.01$, versus control group

\section{Hepatocytes exposed to high glucose increased the expression of $\mathrm{F} 2$}

The reduction of miR-451a was previously observed in GDM patients. To further detect the effect of high glucose on miR-451a, an in vitro high glucose model was constructed. Lower miR451 a levels were observed in hepatocytes exposed to high glucose (Figure $2 \mathrm{~A}$ ). In addition, the mRNA and protein levels of F2 were elevated in high glucose cell model (Figure $2 \mathrm{~B}$ and $\mathrm{C}$ ). Therefore, high glucose levels led to an elevation of $\mathrm{F} 2$ and a reduction of miR-451a.

\section{MiR-451a overexpression inhibited F2 expression}

To explore the possible relationship between miR-451a and F2, we induced miR-451a overexpression in Hep G2 cells. qPCR was performed to verify overexpression efficiency. As shown in Figure $3 \mathrm{~A}$, transfection of miR-451a mimics elevated miR-451a levels. The miR-451a overexpressed cells were subjected to normal or high glucose medium. miR-451a overexpression led to decreased F2 levels. High glucose treatment increased the F2 levels, which were 
abrogated by miR-451a overexpression (Figure 3 $\mathrm{B})$. Moreover, the F2 protein levels yielded a similar pattern (Figure $3 \mathrm{C}$ ). Overall, the results suggest that miR-451a overexpression inhibits F2 expression.
A

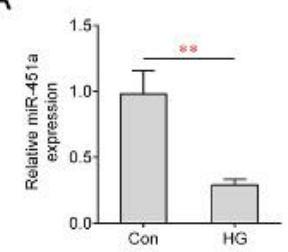

C

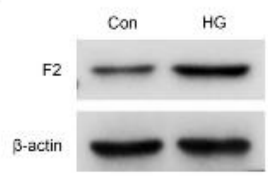

B
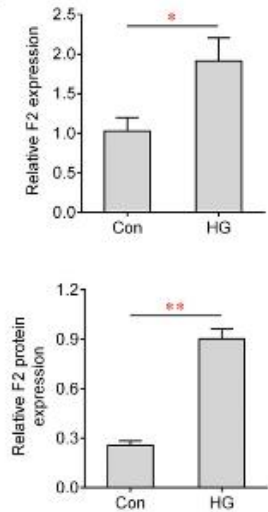

Figure 2: High glucose induces $F 2$ expression in hepatocytes. (A) Relative miR-451a levels in the control and high glucose groups through qPCR assays. (B) Relative F2 levels in control and high glucose groups through qPCR assays. (C) Protein levels of F2 in the control and high glucose groups. *, $p<0.05$ versus control group, ${ }^{* *} p<0.01$ versus control group
A

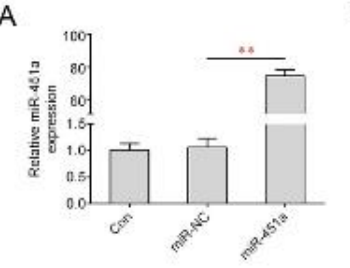

C

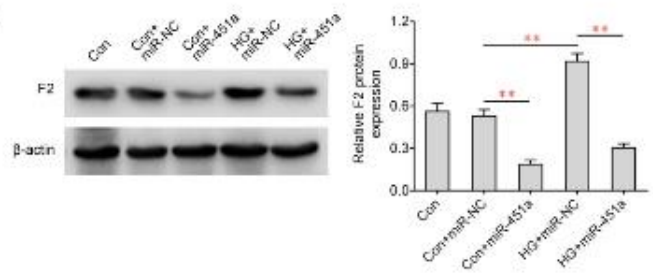

Figure 3: MiR-451a overexpression inhibits F2 expression. (A) Relative miR-451a levels in control or miR-451a transfected groups via qPCR. (B) Relative F2 levels in the control, high glucose and miR-451a transfected groups, or miR-451a transfected and high glucose groups via qPCR. (C) The protein level of F2 in control or high glucose groups. ${ }^{*}, p<0.05$ versus control group. ${ }^{* *} P<0.01$ versus control group

\section{MiR-451a directly targets F2}

Potential targets of miR-451a, were screened for identification through TargetScan (http://www.targetscan.org/vert_72/). The 3'-UTR of F2 harbored a sequence that was complementary to miR-451a, as indicated by bioinformatics analysis (Figure $4 \mathrm{~A}$ ). To confirm the previous data, a luciferase assay was conducted and the results revealed that F2 promoter activity was significantly decreased in miR-451a mimic transfected cells (Figure $4 \mathrm{~B}$ ). However, mutant-type F2 yielded no significant difference in luciferase activity, indicating that F2 is the target of miR-451a. In contrast, miR-451a inhibitor promoted F2 levels (Figure $4 \mathrm{C}$ ). Moreover, F2 protein levels were enhanced in miR-451a inhibitor group (Figure 4 D). Taken together, the data suggest that miR-451a regulates F2 expression.

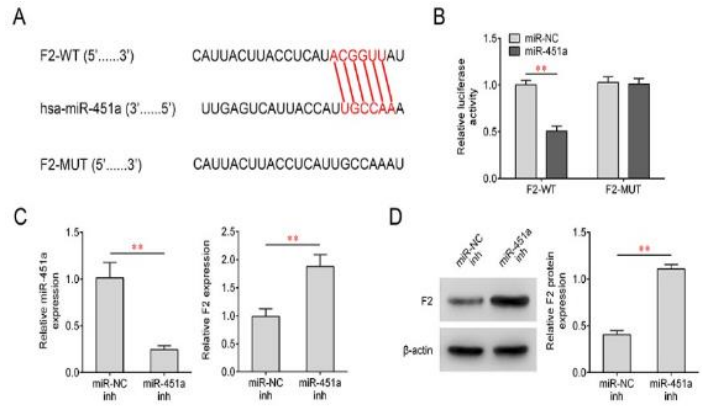

Figure 4: MiR-451a directly targets F2 and inhibits the expression of $F 2$. (A) The predicted binding sites of miR-451a and F2, as well as the mutant sites in F2 MUT. (B) Luciferase assay showed the F2 (WT) and F2 (MUT) promoter activity following transfection of indicated miRNAs. (C) miR-452a and F2 levels were detected after miR-451a inhibitor treatment. (D) F2 protein levels were detected after miR-451a inhibitor treatment. ${ }^{* *} P<0.01$, versus control group

\section{DISCUSSION}

Hypercoagulability, also known as the prethrombotic state, is a complication of gestational diabetes, a maternal and infant health risk, and one of the primary causes of perinatal death [1]. Therefore, detection of changes in blood coagulation degrees in pregnant women with gestational diabetes, as well as the identification of key biomarkers and the development of effective therapeutic drugs, is of great importance in diagnosing and treating gestational diabetes related complications [4]. In this study, blood coagulation was found to be enhanced in patients with GDM, while expression of miR-451a was decreased. These results suggest that miR-451a might be an important biomarker of GDM and can potentially affect the progression of GDM. TEG reflects the whole blood clotting process of thrombus formation and degradation and, consequently, can compensate for deficiencies in traditional blood clotting laboratory examinations. In this study, TEG analysis was performed and coagulation 
differences between GDM and control groups were revealed. TEG can partially reveal the specific characteristics of changes in the coagulation status of pregnant women during pregnancy. Compared with traditional coagulation laboratory tests, TEG is more sensitive and effective in the detection and management of obstetric bleeding.

In addition, high glucose treatment of hepatocytes was found to promote the expression of F2. The role of $\mathrm{F} 2$ in hypercoagulation is already known [12]. Other studies have confirmed the importance of F2 in patients with diabetes $[14,15]$. As was previously reported, prothrombin fragment $F 2$ is a polypeptide fragment produced by the factor $\mathrm{Xa}$ complex after hydrolyzing prothrombin during blood coagulation [13]. This study suggested that the downregulation of miR-451a resulted in an increase of prothrombin expression. As found with miR-451a overexpression assays, the expression of F2 was decreased. Through quantitative PCR assays, immunoblot assays, and luciferase assays, miR-451a was shown to bind to the promoter regions of F2, thereby suppressing the expression of F2. Therefore, the downregulation of miR-451a may promote prothrombin expression.

Previous studies also indicated metabolic abnormalities in GDM patients. For example, in GDM patients, higher concentrations of $\mathrm{HDL}$ were observed in the third trimester of pregnancy, and higher triglyceride levels were found in both the second and third trimesters [14]. Therefore, the hypercoagulable status of GDM patients might be alleviated by targeting miR-451a. The function of miR-451a in a variety of physiological and pathological processes has been widely revealed [15]. For example, MiR451a suppresses the growth and metastasis of papillary thyroid carcinoma cells by suppressing ZEB1 expression [16]. miR-451a is also involved in gastric carcinogenesis via the AKT pathway [17]. Another study showed that miR-451a suppresses the activation of MMP2 and MMP9 in cardiomyocytes upon pathological stress stimulation [18]. In this study, miR-451a suppressed prothrombin expression and contributed to a high blood coagulation state, suggesting a critical role for miR-451a in the physiological regulation of bodies.

\section{CONCLUSION}

An increased coagulation function and decreased expression of miR-451a occur in patients with GDM. In addition, high glucose treatment of hepatocytes promotes the expression of F2. Overexpression of miR-451a inhibits the expression of $\mathrm{F} 2$; therefore, miR451a may be a promising marker for hypercoagulable state GDM patients.

\section{DECLARATIONS}

\section{Conflict of interest}

No conflict of interest is associated with this work.

\section{Contribution of authors}

We declare that this work was done by the authors named in this article and all liabilities pertaining to claims relating to the content of this article will be borne by the authors. Min Qiu designed the study, supervised the data collection, and analyzed the data. Hao Zhang interpreted the data and prepared the manuscript for publication. Zhimin Shangguan supervised the data collection, analyzed the data, and reviewed drafts of the manuscript. All authors read and approved the manuscript.

\section{Open Access}

This is an Open Access article that uses a funding model which does not charge readers or their institutions for access and distributed under the terms of the Creative Commons Attribution License (http://creativecommons.org/licenses/by/ 4.0) and the Budapest Open Access Initiative (http://www.budapestopenaccessinitiative.org/rea d), which permit unrestricted use, distribution, and reproduction in any medium, provided the original work is properly credited.

\section{REFERENCES}

1. Cao M, Zhang L, Chen T, Shi A, Xie K, Li Z, Xu J, Chen Z, Ji C, Wen J. Genetic Susceptibility to Gestational Diabetes Mellitus in a Chinese Population. Front Endocrinol 2020; 11: 247.

2. Yuan $K$, Wang $H$, Chen $Y$, Li S, Wang Q, Cao Y, Gao S, Xu X, Xie Q. A 12-hour comprehensive nutrition care benefits blood glucose level and weight gain and improves outcomes in pregnant women with gestational diabetes mellitus. Ann Palliat Med 2020.

3. Zhu H, He D, Liang N, Lai A, Zeng J, Yu H. High serum triglyceride levels in early first trimester of pregnancy are associated with gestational diabetes mellitus: a prospective cohort study. J Diabetes Invest 2020.

4. You H, Lei A, Xiang J, Wang Y, Luo B, Hu J. Effects of breastfeeding education based on the self-efficacy theory on women with gestational diabetes mellitus: $A$ 
CONSORT-compliant randomized controlled trial. Medicine 2020; 99(16): e19643.

5. Mooney ET, Agostini G, Griebsch C, Hickey $M$. Intravenous vitamin $\mathrm{K} 1$ normalises prothrombin time in 1 hour in dogs with anticoagulant rodenticide toxicosis. Aust Vet J 2020.

6. Ueno El, Fujibayashi K, Sawaguchi J, Yasuda Y, Takano S, Fujioka N, Kawai Y, Fujita H, Tanaka Y, Kajinami K. Monitoring the roles of prothrombin activation fragment 1 and $2(F 1+2)$ in patients with atrial fibrillation receiving rivaroxaban and apixaban. J Thromb Thrombolys 2020.

7. Patti G, Cerchiara E, Bressi E, Giannetti B, Veneri $A D, D i$ Sciascio G, Avvisati G, De Caterina R. Endothelial Dysfunction, Fibrinolytic Activity, and Coagulation Activity in Patients With Atrial Fibrillation According to Type II Diabetes Mellitus Status. Am J Cardiol 2020; 125(5): 751-758.

8. Fornes $D$, Heinecke $F$, Roberti SL, White V, Capobianco $E$, Jawerbaum A. Proinflammation in maternal and fetal livers and circulating miR-122 dysregulation in a GDM rat model induced by intrauterine programming. Mol Cell Endocrinol 2020; 510: 110824.

9. Wang J, Pan Y, Dai F, Wang F, Qiu H, Huang X. Serum miR-195-5p is upregulated in gestational diabetes mellitus. J Clin Lab Anal 2020: e23325.

10. Chen $K$, Zhao XL, Li LB, Huang LY, Tang Z, Luo J, Yang L, Qin AP, Hu F. miR-503/Apelin-12 mediates high glucose-induced microvascular endothelial cells injury via JNK and p38MAPK signaling pathway. Regen Ther 2020; 14: 111-118.

11. Gorbatenko A, Sokilde R, Sorensen EE, Newie I, Persson H, Morancho B, Arribas J, Litman T, Rovira C, Pedersen SF. HER2 and p95HER2 differentially regulate miRNA expression in MCF-7 breast cancer cells and downregulate MYB proteins through miR221/222 and miR-503. Sci Rep 2019; 9(1): 3352.

12. Perez-Boza J, Boeckx A, Lion M, Dequiedt F, Struman I. hnRNPA2B1 inhibits the exosomal export of miR-503 in endothelial cells. Cell Mol Life Sci: CMLS 2020.

13. Panos NG, Cook AM, John S, Jones GM, Neurocritical Care Society Pharmacy Study G. Factor Xa InhibitorRelated Intracranial Hemorrhage (FiX-ICH): Results from a Multicenter, Observational Cohort Receiving Prothrombin Complex Concentrates. Circulation 2020.

14. Liu L, Hu J, Yang L, Wang N, Liu Y, Wei X, Gao M, Wang $Y$, Ma Y, Wen D. Association of WISP1/CCN4 with Risk of Overweight and Gestational Diabetes Mellitus in Chinese Pregnant Women. Dis Markers 2020; 2020: 4934206.

15. Dong $H$, Wang J, Yang $Y$, Zhang $X, Y u M, X u$ G, Jian $P$. Overexpression of miR-451a inhibits cell proliferation by targeted macrophage migration inhibitory factor in HepG2 cells. Chinese J Cell Mol Immunol 2018; 34(12): 1091-1098.

16. Wang Q, Shang J, Zhang Y, Zhou Y, Tang L. MiR-451a restrains the growth and metastatic phenotypes of papillary thyroid carcinoma cells via inhibiting ZEB1. Biomed Pharmac 2020; 127: 109901.

17. Streleckiene $G$, Inciuraite $R$, Juzenas $S$, Salteniene $V$, Steponaitiene R, Gyvyte U, Kiudelis G, Leja M, Ruzgys $P$, Satkauskas $S$ et al. miR-20b and miR-451a Are Involved in Gastric Carcinogenesis through the PI3K/AKT/mTOR Signaling Pathway: Data from Gastric Cancer Patients, Cell Lines and Ins-Gas Mouse Model. Int J Mol Sci 2020; 21(3).

18. Li X, He M, Guo J, Cao T. Upregulation of circular RNA circ-ERBB2 predicts unfavorable prognosis and facilitates the progression of gastric cancer via miR503/CACUL1 and miR-637/MMP-19 signaling. Biochem Bioph Res Co 2019; 511(4): 926-930. 